\title{
Narrativa
}

\section{Il caso Moro nella narrativa ultra-contemporanea}

\section{Ugo Perolino}

\section{OpenEdition}

\section{Journals}

\section{Edizione digitale}

URL: https://journals.openedition.org/narrativa/905

DOI: $10.4000 /$ narrativa.905

ISSN: 2804-1224

\section{Editore}

Presses universitaires de Paris Nanterre

\section{Edizione cartacea}

Data di pubblicazione: 1 décembre 2016

Paginazione: 207-214

ISBN: 978-2-84016-266-7

ISSN: 1166-3243

\section{Notizia bibliografica digitale}

Ugo Perolino, «ll caso Moro nella narrativa ultra-contemporanea», Narrativa [Online], 38 | 2016, online dal 01 janvier 2022, consultato il 22 février 2022. URL: http://journals.openedition.org/narrativa/905; DOI: https://doi.org/10.4000/narrativa.905

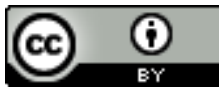

Narrativa est mise à disposition selon les termes de la Licence Creative Commons Attribution 4.0 International. 


\section{Il caso Moro nella narrativa ultra-contemporanea}

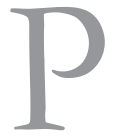

er i suoi effetti sulla memoria degli italiani il caso Moro è stato paragonato all'omicidio di John Kennedy o agli attacchi alle Torri Gemelle dell'11 settembre. Un evento catastrofico, dotato di un potente impatto traumatico, tale da determinare una periodizzazione che separa con forza un "prima" e un "dopo". Percepito o rappresentato immediatamente come il punto di rottura nella continuità storica degli anni Settanta, e forse come il punto terminale di una lunga fase espansiva della democrazia italiana, il sequestro del Presidente della Democrazia Cristiana ha ispirato una fitta biblioteca di narrazioni e mémoires dai più diversi registri stilistici e formali, dalla tragedia alla farsa, scriveva già a caldo Arbasino. Il valore traumatico dell'evento - hanno recentemente ricordato Giancarlo Lombardi e Ruth Glynn - è determinato dalla "lunghezza della prigionia e dal carattere altamente mediatizzato degli eventi" che agiscono ancora nella memoria collettiva come uno "spartiacque", con un "valore metonimico in relazione alla violenza politica degli anni di piombo"1. Il rapimento Moro - è stato altrimenti detto - è la metafora dell'Italia del dopoguerra e del suo fallimento.

In questo contesto non pacificato, dove molte verità sono ancora da accertare, una recente e minuziosa inchiesta giornalistica di Paolo Cucchiarelli ${ }^{2}$ (già

1. Molto utile per la ricchezza di fonti e di materiali consultati l'introduzione, firmata da Giancarlo Lombardi e Ruth Glynn, al volume collettaneo Remembering Aldo Moro. The Cultural Legacy of 1978 Kindapping and Murder, Oxford, Legenda 2012, cui si farà ampiamente riferimento (le citazioni sono a p. 5; mie le traduzioni quando non diversamente indicato). Lombardi e Glynn sottolineano, inoltre, la natura ambigua e aperta dei termini ("caso" e "affaire", entrambi connotati da un senso di eccezionalità nella sfera giudiziaria e politica) utilizzati per indicare il complesso di eventi che hanno luogo tra il 16 marzo e il 9 maggio 1978.

2. Si veda Cucchiareldi, Paolo, Morte di un Presidente. Quello che né lo Stato né le Br hanno mai raccontato sulla prigionia e l'assassinio di Aldo Moro, Firenze, Ponte alle Grazie, 2016. 
autore di Il segreto di piazza Fontana, da cui Marco Tullio Giordana ha tratto il film Romanzo di una strage) riporta l'attenzione su aspetti della vicenda che contrastano con le dichiarazioni rese dai brigatisti e con alcuni aspetti delle ricostruzioni più accreditate. Valorizzando analisi scientifiche non disponibili all'epoca dei fatti, Cucchiarelli si sofferma sulla descrizione del corpo del leader democristiano al momento del ritrovamento in via Caetani: la sabbia sugli indumenti (in particolare un calzino) rivelerebbe che la "prigione del popolo" non coincide con lo stretto cunicolo di via Montalcini, a Roma, ma deve essere forse ricercata in un luogo più vicino al mare; l'analisi balistica delle traiettorie dei proiettili trovati nel corpo e la disposizione dei bossoli all'interno della Renault 4 smentirebbero le testimonianze rese dai brigatisti, che hanno parlato di una raffica diretta contro il prigioniero già disteso nel portabagagli dell'auto.

$\mathrm{Ma}$ Cucchiarelli non è il solo a rimettere in discussione una narrazione ufficializzata da processi, deposizioni, sentenze che si rincorrono nell'arco di quasi quarant'anni. Il lavoro della Commissione Moro, presieduta dall'on. Giuseppe Fioroni, ha riaperto scenari in ombra, rivitalizzato ipotesi precedentemente scartate, suggerito percorsi che attendono di essere valutati con attenzione. Giovanni Pellegrino, già presidente della Commissione stragi nella XI e XII legislatura, è stato ascoltato l'11 novembre 2014. Pellegrino ha ribadito come nel corso del sequestro si sia resa avvertibile una "torsione" nell'atteggiamento dei sequestratori all'altezza del sesto comunicato, quando l'annuncio della fine del processo non fu accompagnato dalla pubblicazione degli interrogatori dell'ostaggio, come i brigatisti avevano promesso. Anche Sciascia aveva notato questa incongruenza, cogliendo nel passaggio alla stampa clandestina l'ammissione di una sconfitta.

"Interessi terzi", che vanno oltre le Br e guardano a uno scenario nel quale agiscono poteri, volontà e soggetti rimasti occulti, sono evocati nelle deposizioni di Gennaro Acquaviva, che nel 1978 era responsabile della segreteria di Bettino Craxi, e di Claudio Signorile (12 luglio 2016), all'epoca dei fatti vicesegretario del partito socialista. Entrambi, Acquaviva e Signorile, hanno confermato la linea di una trattativa portata avanti con interlocutori già noti e individuati nell'area di Potere Operaio, Franco Piperno e Lanfranco Pace, che non portò a sviluppi concreti ${ }^{3}$. Signorile, in particolare, ha ricordato come l'iniziativa socia-

3. Come è noto, Signorile incontrò Franco Piperno attraverso la mediazione di Livio Zanetti, allora direttore dell'Espresso. Il canale di contatto fu aperto da Mario Scialoja, giornalista e militante nell'area di Potere Operaio. Agli incontri, 3 o 4 in tutto, prese parte Lanfranco Pace. 
lista a favore di un negoziato si muovesse in un contesto sfaccettato, dove, accanto alle posizioni della fermezza, coesistevano anche all'interno della Democrazia Cristiana sensibilità attente al dialogo (Bisaglia, Fanfani, Donat Cattin), mentre la galassia dei movimenti extraparlamentari esprimeva posizioni critiche e prese di distanza dall'azione delle Brigate Rosse. Secondo il leader della sinistra socialista le speranze di successo erano legate all'evidenziarsi di una frattura interna al mondo brigatista: "Se dopo i primi quindici giorni Moro non era stato ammazzato", ha raccontato Signorile, "voleva dire che non erano d'accordo"4. Sulla linea della trattativa, resa concretamente operante da una ragnatela di contatti e mediatori, ha insistito Mons. Fabio Fabbri nella sua deposizione (4 febbraio 2016) davanti alla commissione Moro. Fabbri è stato uno stretto collaboratore di Mons. Cesare Curioni, ispettore generale dei cappellani delle carceri. In virtù del suo ruolo, Mons. Curioni poté attivarsi, sollecitato da Paolo VI, per un negoziato volto a ottenere la restituzione di Aldo Moro mediante il pagamento di un riscatto. Secondo Fabbri, che ha raccontato questa vicenda in varie occasioni pubbliche (libri, interviste ecc.), l'emissario del Papa, che aveva contatti con i brigatisti in carcere e con i militanti in libertà, avrebbe incontrato più volte a Napoli un intermediario. Il negoziato era giunto a un punto avanzato, tanto che Fabbri ricorda di avere visto personalmente, a Castel Gandolfo, una ingente quantità di denaro pronta per essere utilizzata per il riscatto di Moro.

Sebbene a distanza di tanti anni sia possibile nutrire dubbi sulla precisione dei ricordi personali e sulla attendibilità di alcune piste, ciò che accomuna le testimonianze che riferiscono delle diverse trattative in atto è il fatto che rendono visibile un'ampia zona grigia la cui esistenza è stata a lungo negata, o almeno rimossa e messa in ombra. Lo scenario descritto non fornisce forse nuovi materiali al plot complottistico, un filone esaurito con l'esaurirsi delle basi ideologiche della guerra fredda, ma sembra erodere i fondamenti veritativi e il consenso pubblico delle narrazioni ufficiali, segnatamente del meccanismo sacrificale, evidenziando un sistema negoziale i cui effetti si diramano sotterraneamente e per canali non visibili.

È questo forse un elemento di differenziazione tra l'elaborazione culturale interna ai confini nazionali - dove riprende quota un filone di accertamenti e di

4. La registrazione delle audizioni della "Commissione parlamentare di inchiesta sul rapimento e sulla morte di Aldo Moro", presieduta dall'on. Giuseppe Fioroni (tra i commissari figurano esperti e studiosi della vicenda Moro come Miguel Gotor), sono disponibili sul sito di Radio Radicale. 
inchieste - e la direzione generale delle ricerche degli italianisti che operano all'estero, soprattutto nel mondo anglosassone, dove una lunga tradizione - si pensi a The Moro Morality Play. Terrorism as Social Drama (1986) di Robin Erika Wagner-Pacifici - utilizza chiavi di lettura derivate dai modelli antropologici per inquadrare gli anni di piombo e i fatti legati al terrorismo italiano (e in parte anche alle narrazioni di mafia).

Un esempio di elegante incisività teorica è fornito dal lavoro di Pierpaolo Antonello, il quale applica plasticamente al caso Moro la teoria girardiana del capro espiatorio ${ }^{5}$. Il registro ermeneutico del sacrificio, ha notato Antonello, esprime in primo luogo la consapevolezza delle vittime - "the sacrificial narrative is above all the progressive awareness of being a subject of a scapegoat mechanism, of a vicitimary persecution"'. Accomunati da un destino di vittime, Pasolini e Moro leggono la loro condizione attraverso un paradigma cristologico e forniscono a scrittori e registi che si occuperanno del loro caso una cornice interpretativa nella quale disporre gli eventi. Per marcare le differenze rispetto ad altre modalità di emplotment occorre inoltre rilevare l'antitesi tipologica per cui, da un lato, la narrazione sacrificale integra lo schema edipico, ponendo l'accento sulla dimensione sociale e antropologica piuttosto che sull'inconscio, mentre dall'altro lato decostruisce "the narrative closure of conspiracy theory" , basata sulla falsa illusione di una interpretazione coerente e sistematicamente compiuta dei fatti storici.

La produzione narrativa ultra-contemporanea in Italia ha registrato la svolta post-sacrificale con una sottile volontà di denunciare l'iterazione retorica delle rappresentazioni convenzionali del passato. L'efficacia degli apparati simbolici - il caso Moro come spartiacque - appare dunque depotenziata, e se conserva un grado ancora registrabile di immanenza, risulta declinata nell'universo formale della riscrittura, con le connesse figure delocalizzanti (l'ironia, il metalinguaggio) che rimettono in discussione i codici dell'ufficialità, le formule celebrative, le verità accreditate. "Aldo è morto, e non me ne sono neanche accorto" recita il monologo di Michele Timpano dal titolo (irriverente) Aldo Morto, una rutilante parodia dei discorsi celebrativi, uno scintillante sberleffo rivolto alla imbalsamazione funeraria della Prima Repubblica.

5. Si veda Antonello, Pierpaolo, "Narratives of Sacrifices: Pasolini and Moro", in Antonello, Pierpaolo, O'Leary, Alan, Immagining Terrorism: The Rethoric and Representation of Political Violence in Italy 1969-2009, Oxford, Legenda, 2010, pp. 30-47.

6. Ibid., p. 31.

7. Ibid. 
Se si guarda alla quantità di fiction costruita attorno al sequestro Moro (romanzi, racconti, serie televisive, film, opere teatrali) si ha la conferma che quel set di eventi costituisce un nucleo solido interessato da un defatigante processo di riscrittura. Alan O'Leary ha opportunamente richiamato la metafora del "palinsesto" a proposito del doppio finale di Buongiorno, notte, il film di Bellocchio del $2003^{8}$. Nel primo caso la storia si conclude con la morte dell'ostaggio, come nella realtà storica; in un'altra sequenza, onirica, Moro cammina libero, nella luce del primo mattino, ancora vivo e fuori dalla prigione di via Montalcini. Il film di Bellocchio, ha notato O'Leary, non propone soltanto una lettura degli eventi legati al sequestro, quanto piuttosto una riflessione sui mezzi di rappresentazione, sulle modalità di percezione e racconto attraverso le quali questi eventi sono stati culturalmente costruiti e stabilizzati nella memoria pubblica. Siamo nei pressi di quella definizione di finzioni metastoriche utilizzata di recente da Claudia Boscolo e Stefano Jossa per indicare “un'esperienza narrativa che dalla storia parte ma attraverso la storia si interroga sulle proprie possibilità di senso e sul proprio statuto di verità"’. Il doppio finale, del resto, appartiene alla tradizione della parodia: non si trova per la prima volta in Buongiorno, notte, dal momento che Bellocchio è stato anticipato da Giampaolo Rugarli. Nel romanzo La troga, pubblicato da Adelphi dieci anni dopo la strage di via Fani, Rugarli immagina che l'onorevole Lauro Grato Sabbioneta, palese controfigura di Moro, partecipi attivamente all'organizzazione del proprio sequestro per inscenare fittiziamente la propria morte. Un inganno che gli consente di allontanarsi dal mondo corrotto e violento della politica, ormai intrecciato con la criminalità organizzata.

La prospettiva di Rugarli è però antifrastica, discende dalla satira di Arbasino piuttosto che dalla linea tragica e sacrificale di Sciascia ${ }^{10}$, e in questo senso è apparsa a lungo come una linea minoritaria, piuttosto appartata al confronto della monumentalizzazione del sacrificio come momento chiave per la soluzione della crisi degli anni di piombo. L'altra faccia del meccanismo vittimario,

8. Per un quadro generale del caso Moro al cinema si veda O’LeAry, Alan, Tragedia allitaliana. Cinema e terrorismo tra Moro e memoria, traduzione di Angelica Salaris, Città di Castello, Angelica, 2007.

9. Si veda Boscolo, Claudia, Jossa, Stefano, Finzioni metastoriche e sguardi politici dalla narrativa italiana contemporanea, in AA.VV., Scritture di resistenza, Roma, Carocci, 2014, pp. 15-67.

10. Per un approfondimento di questo aspetto si veda Perolino, Ugo, "La cripta e il fantasma. Narrazioni e memorie degli anni di piombo in Baliani, Pieri, Delbono", in Casalino, Leonardo, Cedola, Andrea, Perolino, Ugo (a cura di), Il caso Moro. Memorie e narrazioni, Massa, Transeuropa, 2016, pp. 27-47. 
del resto, è la spettralizzazione, il ritorno del rimosso, la messa in scena di un contenuto ancora informe, una verità da riesumare e da sciogliere. La riduzione di Moro a spettro è praticata, tra ironia decostruttiva e tedio, con una punta di fastidio per la natura colloidale di ciò che resta della prima Repubblica, da Ferruccio Parazzoli11 ${ }^{11}$ e ancora più esplicitamente da Alessandro Banda ${ }^{12}$.

Ferruccio Parazzoli maneggia con assoluta asciuttezza di stile la materia narrativa di un fantasy teologico; il caso Moro rivela i termini di un gioco, una scommessa tra Dio e Satana, nell'eterna lotta tra il bene e il male. Verso la fine del romanzo Satana porta Moro fuori della prigione, in alto sull'Altare della Patria, e gli mostra l'ombra enormemente invecchiata di Giulio Andreotti, unico sopravvissuto di quella stagione della Repubblica. Moro allora interroga quell'ombra, la prega di narrargli quel futuro che a lui non è concesso di vivere, gli si rivolge con la residua speranza che il suo sacrificio non sarà stato inutile. Quella che segue è una visione sincopata dei decenni che sarebbero venuti, segnati da stragi di mafia e delitti politici, un corteo di fantasmi. Infine, come a incarnare una previsione inscritta in una delle sue lettere più risentite e accorate, nell'ultima sequenza Moro torna in Parlamento, si aggira come un'ombra tra i banchi dei deputati, un corpo sanguinante crivellato di colpi.

Anche Alessandro Banda (classe 1963) si serve di un registro non ideologico, decantato, venato di incredulità, animato da un atteggiamento reattivo alla museificazione di fatti ormai storici che appaiono come sequestrati, anchilosati, incapsulati in pròtesi di memoria. Lo spostamento dell'azione in un contesto medianico (a parlare sono le voci dei trapassati) è collegato al senso di saturazione dei segni: "Tre personaggi illustri, così noti che il loro nome ha finito per usurarsi e non è più citabile"13. La non citabilità dei nomi è il risultato di un eccesso, un abuso del linguaggio, ma esclude anche, per ammissione dell'autore, ogni ipotesi postmodernistica di riscrittura o double coding: "Sapevo, sulla base della mia pluriennale esperienza", racconta il Moro ultraterreno e spettralizzato di Banda, "che, nel dibattito politico, le parole non contano affatto per se stesse, per il loro significato effettivo. Esse contano per il suono, magico. Formule magiche"14. Moro, Pasolini, Lady D: nell'ordine, un Presidente, un Poeta, una Principessa, interrogati sul mistero che affligge "la fine delle loro esperienze

11. Parazzoli, Ferruccio, Adesso viene la notte, Milano, Mondadori, 2008, e Altare della Patria, Milano, Il Saggiatore, 2011.

12. BANDA, Alessandro, Come imparare a essere niente, Parma, Guanda, 2010.

13. Ibid., p. 10.

14. Ibid., pp. 37-38. 
terrene". "Voci scorporate", dunque, capaci di dialogare mentre il peso delle identità che abitarono "pare irrimediabilmente evaporare nell'indistinto" Nella sua ricostruzione dei fatti l'ombra di Moro ironizza sui suoi sequestratori, ritenuti incapaci di portare a termine un'operazione di questo genere senza l'ausilio di altri professionisti del crimine e della guerriglia ${ }^{16}$. La strage di via Fani è preceduta da oscuri presagi, simili a quelli che nel teatro tragico precedono l'ora di sangue delle Idi di Marzo. Attraverso la voce d'oltretomba dello statista l'autore avalla la tesi degli "interessi terzi" e della collaborazione al piano brigatista di altre e più micidiali "entità", ma qualifica al tempo stesso l'intera ricostruzione come una struttura retorica, un'altra possibile forma del dire dentro uno spazio linguistico imploso, mentre la logica degli avvenimenti, la realtà effettuale, è dominata dal caso e preda dell'imprevedibile: "Fui scelto per caso, ecco la verità. Mi cascarono le braccia ma andò così, e il caso non sbaglia. A rovinarmi fu la frezza, il ciuffo bianco che spiccava in mezzo ai miei capelli grigi" ${ }^{17}$. Ma se ogni narrazione è costituzionalmente insincera, dal momento che abroga le contingenze e istituisce un regime di motivazioni linguistiche dove vige la pura casualità, la sola via per reagire all'eterno ritorno della menzogna e alla torturante embolia della parola consiste appunto nell'imparare a essere niente.

La spettralizzazione di Moro, e non soltanto in un'accezione metaforica, era già avvertita da Adriano Sofri in un libro, L'ombra di Moro (1991), scritto a ridosso del ritrovamento delle nuove carte del Memoriale nell'appartamento di via Monte Nevoso a Milano:

I dodici anni di cui c'è stato bisogno per completare la perquisizione di un appartamento-covo sono bastati ad allungare senza riguardi l'elenco di quelli che sono andati al di là. Moro ha preceduto solo di pochissimo quel papa Paolo VI che si è presentato al Padre Eterno - nella cui fede, pare, vacillava angosciosamente, tratto mirabile della sua figura - con l'amaro viatico dell'invettiva pronunciata in Laterano. È morto Carlo Alberto Dalla Chiesa, sono morti Enrico Berlinguer e Benigno Zaccagnini, è morta Elsa Morante, Italo Calvino e Alberto Moravia, è morto Leonardo Sciascia ${ }^{18}$.

15. Ibid., p. 11.

16. I quattro brigatisti vestiti da avieri, responsabili diretti dell'agguato e della strage di via Fani, sono in realtà spaventati e impreparati, le armi si inceppano, mentre l'efficacia dell'azione armata è garantita da due "tiratori scelti" non ulteriormente identificati.

17. BANDA, Alessandro, Come imparare a essere niente, cit., p. 23.

18. Sofri, Adriano, L'ombra di Moro, Palermo, Sellerio, 1991, p. 74. 
Una lista che intanto si è allungata: Cossiga, Andreotti, Gallinari. Scriveva Sofri al principio degli anni Novanta: "Mi appare chiaro, oggi più che nel 1978, che nella morte di Moro buona parte della classe pubblica italiana ha cercato di venire a patti con la propria morte" 19 .

Il primo a tracciare un parallelo tra Moro e Pasolini è stato forse Leonardo Sciascia, ripreso in questo (e altro) da Sofri che ha notato come alcuni luoghi del Memoriale richiamino stranamente certi passaggi degli Scritti corsari. Mi pare che questa coincidenza di toni e qualche volta di parole non sia stata molto osservata, mentre di recente il parallelo Moro-Pasolini è stato ripreso da Pierpaolo Antonello. Il nucleo di questa interpretazione è preso dalla teoria girardiana: la morte di un uomo innocente viene sfruttata dal potere politico per ristabilire la "ragione di stato" e riaffermare la coesione dell'ordine sociale scompaginato da una crisi endogena di odio e violenza generalizzata. Nel modello girardiano adottato da Antonello il caso Moro presenta le caratteristiche eziologiche del capro espiatorio, soprattutto per l'unanimità che informa il processo di selezione vittimaria e di espulsione sacrificale ${ }^{20}$. L'immaginario appartiene alla conoscenza storica, e la modifica, nel momento in cui l'oggettività dei fatti è disposta in un ordine sequenziale e nel sistema di referenze di una grammatica simbolica. Quando Sciascia, a caldo, sottolineava come l'affaire Moro "vivesse già tutto in letteratura", la sua osservazione evidenziava questo mimetismo originario che dà forma al racconto ${ }^{21}$. Questa "affinità" rende il caso Moro disponibile all'esegesi letteraria (particolarmente in tempi di "nuovo realismo") e fissa storicamente l'acme degli anni di piombo nello schema tragico della morte del re. Al tempo stesso, come si è visto, proprio alla decostruzione del modello sacrificale si sono dedicate narrazioni come quelle di Parazzoli e Banda, attraverso un sistema citazionistico e parodistico che corrode verità ormai instabili.

Ugo Perolino

Università G. d'Annunzio di Chieti-Pescara

19. Ibid., p. 76.

20. Si veda Antonello, Pierpaolo, "Narratives of Sacrifice: Pasolini and Moro", cit., p. 31.

21. Osserva infatti Antonello che il caso Moro "seemed to present itself as an archetypical event that world literature had already narrated in many versions and variants", ibid., pp. 37-38. 